\title{
The He/Ne beam diagnostic for line-ratio spectroscopy in the island divertor of Wendelstein 7-X
}

\author{
T. Barbuia, S.A. Bozhenkov ${ }^{b}$, F. Effenberga, C. Favreauc, E. Flom ${ }^{a}$, G. Fuchert ${ }^{b}$, P. \\ Drews $^{\mathrm{e}}$, K. Hammond ${ }^{\mathrm{b}}$, M. Jakubowski ${ }^{\mathrm{b}}$, C. Killer ${ }^{\mathrm{b}}$, R. König ${ }^{\mathrm{b}}$, M. Krychowiak ${ }^{\mathrm{b}}$,'S. \\ Loch $^{c}$, J. Muñoz Burgos ${ }^{d}$, E. Pasch ${ }^{b}$, O. Schmitz $^{\mathrm{a}}$, E.R. Scott ${ }^{\mathrm{b}}, \mathbf{M}$. Vecsei ${ }^{\dagger}, \mathbf{S}$. \\ Zoletnik $^{\dagger}$ and the W7-X Team ${ }^{b}$ \\ ${ }^{a}$ University of Wisconsin, Madison WI, USA \\ ${ }^{b}$ Max-Planck-Institut für Plasmaphysik, Greifswald, Germany \\ ${ }^{c}$ Auburn University, Auburn AL, USA \\ ${ }^{d}$ Astro Fusion Spectre LLC, San Diego CA, USA \\ ${ }^{e}$ Forschungszentrum Jülich GmbH, Institut für Energie- und Klimaforschung - Plasmaphysik, Partner \\ of the Trilateral Euregio Cluster (TEC), Jülich, Germany \\ ${ }^{f}$ Wigner Research Centre for Physics, Budapest, Hungary
}

\section{E-mail: barbui@wisc.edu}

ABSTRACT: a versatile gas injection system and an absolute calibrated spectroscopic observation system with more than 200 lines-of-sight have been installed in two divertor locations in the Wendelstein 7-X stellarator. This powerful setup enables among other measurements to infer $T_{\mathrm{e}}$ and $n_{\mathrm{e}}$ in the divertor region by means of line-ratio spectroscopy on thermal helium. This is a robust and routinely used diagnostic at W7-X and this work describes its main characteristics and shows some examples of $T_{\mathrm{e}}$ and $n_{\mathrm{e}}$ profiles. The applicability of the diagnostic is currently being extended towards the low $T_{\mathrm{e}}$ and high $n_{\mathrm{e}}$ conditions occurring during detachment by means of line-ratio spectroscopy on neon. Initial $T_{\mathrm{e}}, n_{\mathrm{e}}$ profiles obtained with a neon-beam are here presented.

KEYWORDS: line-ratio spectroscopy; collisional-radiative model; 


\section{Contents}

\section{Introduction}

\section{Experimental setup}

2.1 The gas injection system

2.2 The observation system

\section{Experimental results}

3.1 Helium line-ratio spectroscopy

3.2 Neon line-ratio spectroscopy

\section{Introduction}

Line-ratio (LR) spectroscopy on helium is widely used in fusion devices as a diagnostic to measure electron temperature and density profiles in the scrape-off-layer and the plasma edge [1] [2]. It is based on the density and temperature dependence of the ratio of selected emission lines of neutral He. At Wendelstein 7-X (W7-X) LR on thermal He is routinely used to infer radial profiles of $T_{\mathrm{e}}$ and $n_{\mathrm{e}}$ in the divertor region in the attached divertor conditions. In the low temperature $(<<10 \mathrm{eV})$ and high density $\left(>10^{20} \mathrm{~m}^{-3}\right)$ conditions occurring in the $\mathrm{W} 7-\mathrm{X}$ divertor during detachment, He lines are weak (or not detectable for $T_{\mathrm{e}}<5 \mathrm{eV}$ ) and $T_{\mathrm{e}}, n_{\mathrm{e}}$ measurement suffers from great uncertainties. In order to overcome this limitation, LR spectroscopy can be performed on $\mathrm{Ne}$ instead of $\mathrm{He}$, or in a $\mathrm{Ne}+\mathrm{He}$ gas mixture [3]. Ne has lower excitation energy and can provide stronger emission lines at low temperatures.

In this work the divertor gas injection system and the divertor visible spectroscopy observation system are described. Examples of radial profiles of electron temperature and density obtained through He LR spectroscopy are shown and compared with other edge diagnostics. Two He collisional-radiative models with a different number of considered atomic levels are employed to infer $T_{\mathrm{e}}, n_{\mathrm{e}}$ from the line-ratios and the different results are discussed.

First attempts to perform LR spectroscopy on neutral neon have been carried out in the first and second island divertor campaigns (2017-2018). Injections of pure $\mathrm{Ne}$ and mixed $\mathrm{Ne}+\mathrm{He}$ have been performed to extent the applicability of the diagnostic to the detached divertor regime. Initial candidates for LR on Ne have been determined and first $T_{\mathrm{e}}, n_{\mathrm{e}}$ profiles are obtained and compared to $T_{\mathrm{e}}, n_{\mathrm{e}}$ profiles from $\mathrm{He} \mathrm{LR}$.

\section{Experimental setup}

\subsection{The gas injection system}


The gas injection system is provided by two in vacuo plugins consisting of gas and water lines as well as a gas box [4]. In the gas box five independent piezo valves allow the gas injection to be carried out at different poloidal positions [5]. Poloidal separation of each valve is $2.5 \mathrm{~cm}$. One valve for each gas box is connected to a fast power supply allowing an opening time of $\sim 2$ $\mathrm{ms}$. The other valves have an opening time of $\sim 12 \mathrm{~ms}$. All valves are equipped with thin capillary nozzles with inner diameter of $0.6 \mathrm{~mm}$ and length of $\sim 10 \mathrm{~cm}$ for small beam divergence.

The gas boxes are mounted at a position directly behind the divertor plates in two divertor modules, one lower divertor module (machine module HM30) and one upper divertor module (HM51), which are connected by the same magnetic flux tube in the $m / n=5 / 5$ standard island divertor configuration of W7-X. The vacuum plugins are water cooled and with this capable of steady-state plasma operations. This system allows puffing different gases for many purposes: $\mathrm{He}$ and $\mathrm{Ne}$ for emission spectroscopy, Ar for impurity transport studies, $\mathrm{Ne}$ and $\mathrm{N}_{2}$ for edge radiative cooling experiments, $\mathrm{H}_{2}$ for fueling with e.g. density feedback, $\mathrm{CH}_{4}$ for quantifying the $\mathrm{C}$ source. The gas reservoir pressure ranges from few mbar up to 60 bar. For the purpose of emission spectroscopy the reservoir pressure is set to $50 \mathrm{mbar}(\mathrm{He})$ or $30 \mathrm{mbar}(\mathrm{Ne})$ corresponding to a flow rate of about $5 \times 10^{18} \mathrm{a} / \mathrm{s}$ : high enough to detect the light from line emissions but low enough not to affect the global and local plasma parameters. The thermal beam has a mean velocity of $1.5 \mathrm{~km} \mathrm{~s}^{-1}$ and a divergence of $\sim 40^{\circ}$.

\subsection{The observation system}

The observation system of the divertor spectroscopy is realized in both modules with two arrays of 54 lines-of-sight $(\mathrm{LoS})$ each in the radial and poloidal direction. Figure 1 presents an overview of the observation system in the upper divertor module. In the radial direction the LoS have an extension of $13.5 \mathrm{~cm}$ with a spatial resolution of 2-3 $\mathrm{mm}$. They cover the full radial width of the SOL and few $\mathrm{cm}$ of the confined region. In the poloidal direction the LoS have an extension of $60 \mathrm{~cm}$ with a spatial resolution of 10-20 mm. They cover almost the full width of the horizontal and vertical divertor targets. The LoS are defined by an array of optical fibers (core diameter $=$ $165 \mu \mathrm{m}$, length $=\sim 80 \mathrm{~m}$ ) fitted to a UV achromatic lens mounted directly behind a vacuum window at a diagnostic observation port. The optical fibers and the optics for the poloidal observation are installed inside an immersion tube.

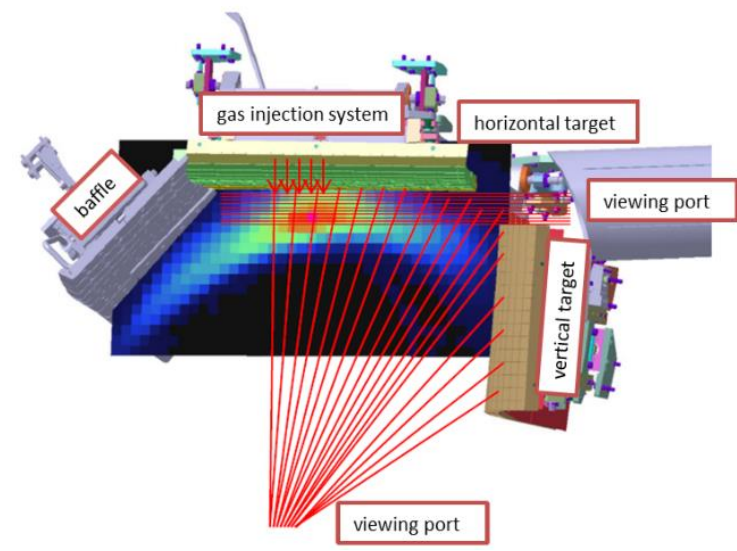

Figure 1. Overview of the observation system for the divertor spectroscopy in the upper divertor of W7-X with indicated lines of sight viewing from two observation ports. The red arrows represent the five separated 
nozzles. The colored area represents a sample EMC3-EIRENE simulation of the He line emission $(\lambda=$ 706.5) [6].

The light from the fibers is guided to several spectrometers installed in a laboratory outside of the torus hall. The fibers are fitted to the spectrometer's entrance slit. The radial LoS in HM30 are channeled to a $20 \mathrm{~cm}$ and a $32 \mathrm{~cm}$ focal length Czerny-Turner spectrometer. The poloidal LoS in HM30 are channeled to a $20 \mathrm{~cm}$ and a high resolution SOPRA spectrometer with a focal length of $1.5 \mathrm{~m}$. The radial LoS in HM51 are channeled to a $20 \mathrm{~cm}$ and a $75 \mathrm{~cm}$ focal length CzernyTurner spectrometer. The poloidal LoS in HM51 are channeled to a $20 \mathrm{~cm}$ and a $32 \mathrm{~cm}$ focal length Czerny-Turner spectrometer. At the spectrometer exit, the light is detected by a CCD camera with a frame transfer EMCCD sensor with $1024 \times 1024$ pixels $(13 \times 13 \mu \mathrm{m}$ each) [7]. It is normally used in $1024 \times 27$ binning mode, $5 \mathrm{MHz}$ ADC rate, providing acquisitions with a time resolution of 25 $\mathrm{ms}$. The dispersion of the system varies from $0.4 \mathrm{~nm} / \mathrm{pxl}$ down to $1 \mathrm{pm} / \mathrm{pxl}$, depending on the spectrometer and grating. All systems have been intensity calibrated with an integrating (Ulbricht) sphere. This spectroscopic system allows to observe the emissions from the divertor region over a broad wavelength range, from near UV to near IR $(350$ - $900 \mathrm{~nm})$, with high spectral resolution. It permits to study different topics by observing specific emissions: determining $T_{\mathrm{e}}$ and $n_{\mathrm{e}}$ from the ratio of selected lines, particle density and fluxes from the line intensity, ion impurity temperature from the line broadening, particle bulk drifts from Doppler line shift, magnetic field strength from Zeeman splitting, spectroscopic molecule analysis, wall conditioning analysis, and others.

He-beam LR spectroscopy is normally carried out using the bottom divertor setup (HM30) employing $27+27$ of the total LoS in radial and poloidal direction, channeled to two $20 \mathrm{~cm}$ focal length Czerny-Turner spectrometers. They are equipped with a $600 \mathrm{gr} / \mathrm{mm}$ grating and observe the three $\mathrm{He}$ I lines with a dispersion of $0.1 \mathrm{~nm} / \mathrm{pxl}$. In the radial direction this setup allows to measure the He lines in front of the used nozzle for the full radial width of the SOL. In the standard magnetic configuration this width is $\sim 9 \mathrm{~cm}$. Beyond the last closed flux surface helium is ionized. In the poloidal direction $\mathrm{He}$ I emissions from the beam are typically visible in 6 channels in the vicinity of the nozzle, thus covering a poloidal extension of $\sim 13 \mathrm{~cm}$.

\section{Experimental results}

\subsection{Helium line-ratio spectroscopy}

Repetitive (up to 20) 75 ms-long helium puffs are carried out during the discharge through one or multiple nozzles in series from the lower gas box. Spectra covering the region 650-750 nm are acquired continuously during the discharge with an exposure time of $25 \mathrm{~ms}$. The measured He I lines are the singlet $\lambda_{1}=667.8$ and $\lambda_{2}=728.1 \mathrm{~nm}$ as well as the triplet $\lambda_{3}=706.5 \mathrm{~nm}$ visible lines. Subtracting the background (beam off) and multiplying by the calibration factors allows to obtain the absolute value of the line intensities. A nonlinear least square Gaussian fit is then applied to the lines and its integral gives the line intensity.

From these line intensities two ratios are computed: $I_{706} / I_{728}$ which mainly depends on $T_{\mathrm{e}}$, and $I_{668} / I_{728}$ which is sensitive to $n_{\mathrm{e}}$. The derivation of $T_{\mathrm{e}}$ and $n_{\mathrm{e}}$ is done by comparing these experimental ratios with synthetic ratios calculated using a collisional-radiative model (CRM). This model simulates the spectral emission of the neutral He taking into account the significant populating and depopulating mechanisms for the considered atomic levels. The present version 
of the model accounts for spontaneous decay, electron-impact excitation/de-excitation, electronimpact ionization. Ion-impact, charge-exchange and recombination processes are negligible in the helium thermal beam case and therefore excluded from the model [8].

The successful application of the line-ratio technique depends strongly on the accuracy of the set of atomic data used in the CRM. In this work we use a hybrid time dependent/independent CRM with a renewed set of atomic data [8]. This model takes into account the long relaxation time of the $2^{3} S$ metastable term of helium that populates the triplet spin system. The time dependent solution is important only at low densities $\left(<5 \times 10^{18} \mathrm{~m}^{-3}\right)$ at which the relaxation times for the metastable-triplet system $(\sim 10 \mu \mathrm{s})$ are comparable to the beam propagation time. This CRM exists as a data set that accounts for the first 19 levels of the neutral helium (up to $n=4$ ) and treats every higher excitation as ionization. The same model exists also as a data set that includes contributions from high Rydberg states up to $n=500$ by means of a projection matrix which projects the high $n$-shell effects into the lower 19-term population [9]. This inclusion has a significant effect at high densities and temperatures. There is no direct way to investigate experimentally the effects of the high Rydberg states onto the lower populations. Previous works based on this CRM with an earlier version of the atomic data have shown that the data set which gives the best agreement in terms of $T_{\mathrm{e}}$ and $n_{\mathrm{e}}$ with other edge diagnostics depends on the investigated $T_{\mathrm{e}}$ and $n_{\mathrm{e}}$ range as well as on the chosen line-ratio [8] [2]. Hence it is fundamental to compare $T_{\mathrm{e}}$ and $n_{\mathrm{e}}$ determined using both data sets to other edge diagnostics to find out which one gives the best agreement. In this work $T_{\mathrm{e}}$ and $n_{\mathrm{e}}$ are estimated from He LR using both data sets and the results are compared with other edge diagnostics available at W7-X, specifically these are the Langmuir probe mounted on a reciprocating manipulator [10] [11], the Alkali-metal beam [12], the edge channels of the Thomson scattering system [13] and the divertor Langmuir probes [14]. Two discharges at middle heating power $\left(\mathrm{P}_{\mathrm{ECRH}}=4 \mathrm{MW}\right)$ and low density $\left(\bar{n}_{\mathrm{e}}=4 \times 10^{19} \mathrm{~m}^{-3}\right)$ are selected in two different magnetic configurations. Figure 2 shows $T_{\mathrm{e}}$ and $n_{\mathrm{e}}$ profiles from the different diagnostics for a standard magnetic configuration where the $5 / 5$ island is present in the SOL. The presence of the island causes the $T_{\mathrm{e}}$ profile to have a local minimum and the $n_{\mathrm{e}}$ profile to be flat inside the island. Figure 3 shows the profiles for a high iota limiter configuration with no islands in the SOL. Both $T_{\mathrm{e}}$ and $n_{\mathrm{e}}$ profiles are in this case monotonically decaying from the LCFS to the far SOL.
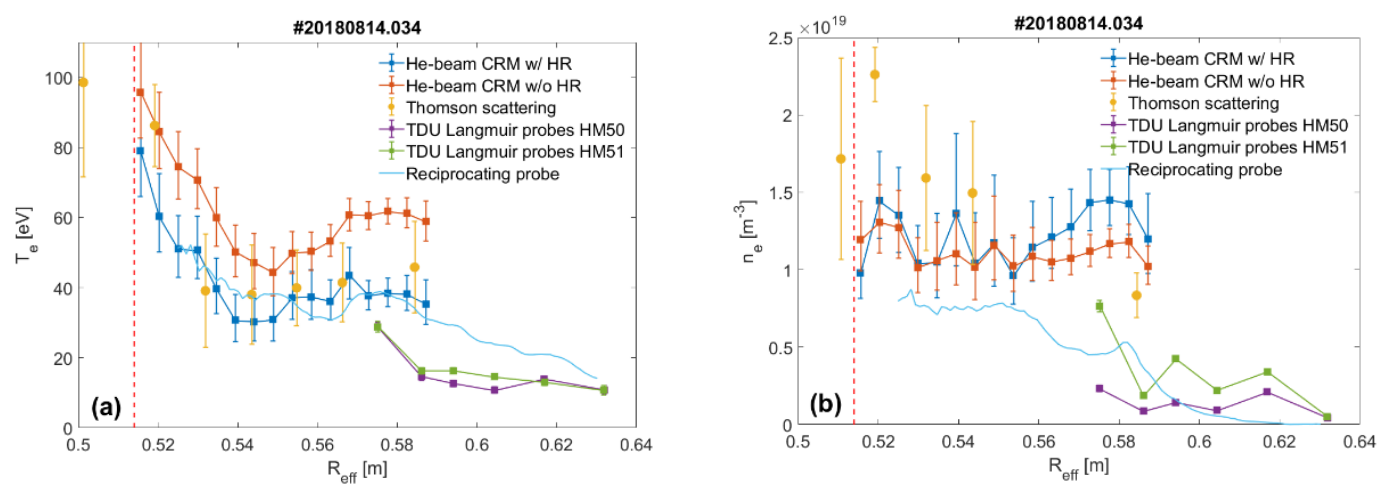

Figure 2. Radial profiles of $T_{\mathrm{e}}(a)$ and $n_{\mathrm{e}}(b)$ determined with the He-beam diagnostic with and without inclusion of the high Rydberg states compared with other edge diagnostics. Standard magnetic configuration. LCFS is indicated with a vertical red dashed line. 

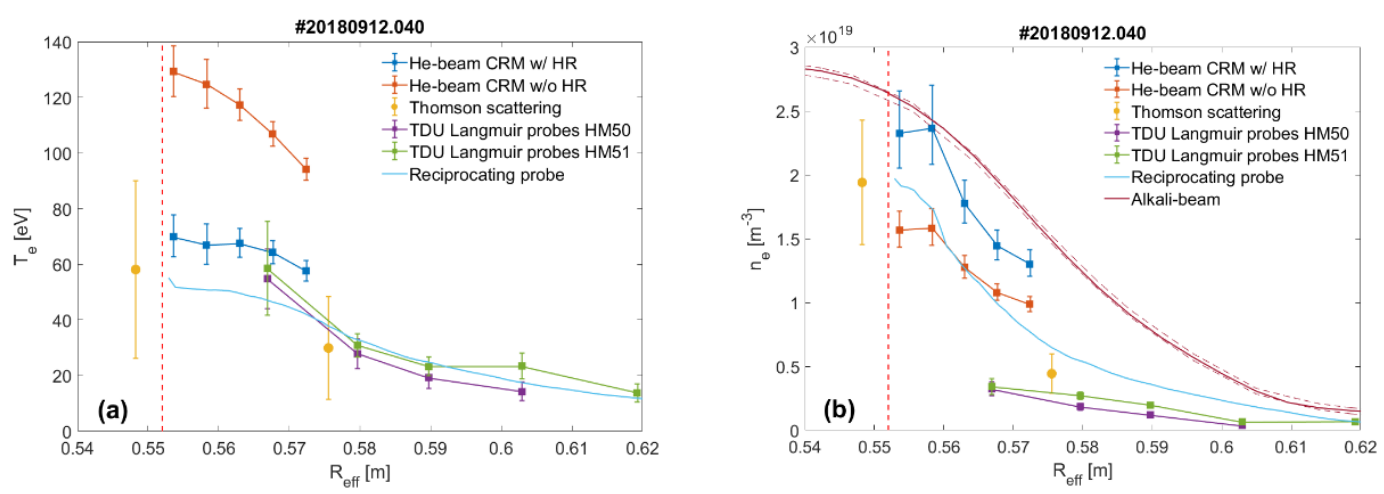

Figure 3. Radial profiles of $T_{\mathrm{e}}(a)$ and $n_{\mathrm{e}}(b)$ determined with the He-beam diagnostic with and without inclusion of the high Rydberg states compared with other edge diagnostics. High iota limiter configuration. LCFS is indicated with a vertical red dashed line.

Comparing the $T_{\mathrm{e}}$ and $n_{\mathrm{e}}$ radial profiles obtained with the He-beam to other edge diagnostics we observe that there is a large difference in the temperatures estimated with and without inclusion of the high Rydberg states, especially at high densities. The CRM with inclusion of high Rydberg states gives temperatures which agree with the other edge diagnostics, while the CRM without high Rydberg states overestimates $T_{\mathrm{e}}$ especially at high densities, delivering $T_{\mathrm{e}}$ up to a factor of 2 higher than the model with high Rydberg states. Regarding the density estimation the two CRMs deliver similar results at medium/low densities $\left(<=1 \times 10^{19} \mathrm{~m}^{-3}\right)$. At high densities $\left(>1.5 \times 10^{19} \mathrm{~m}^{-3}\right)$ the results from the two CRMs differ. The CRM without inclusion of high Rydberg gives lower $n_{\mathrm{e}}$ while the model with inclusion of high Rydberg states gives higher $n_{\mathrm{e}}$. Given the spread of density values measured by the other edge diagnostics it is not possible to state which model gives the best agreement. Both estimates lie within the ranges determined by the other measurements.

In conclusion the discrepancy between the two models is evident especially at high temperatures and densities, originating from the different number and treatment of the Rydberg states. It is believed that the fact that the model with no high Rydberg states considers only the levels up to $n=4$ is partially the reason of the deviation from the model with high Rydberg states included. The model with inclusion of high Rydberg states is to be preferred to the one without, since it provides $T_{\mathrm{e}}$ that better agrees with other diagnostics. Nevertheless, as already pointed out in [2], one might have to assume that dependent on the electron density different treatments of the high Rydberg states may be necessary, especially because highly excited Rydberg states in the high $n_{\mathrm{e}}$ range may be physically impossible [8]. Moreover the cut-off level of the included high Rydberg states is also a matter of discussion. $n=500$ might be too high, in fact another study based on a different CRM suggests to stop at $n=26$ [15].

The uncertainties for the $T_{\mathrm{e}}, n_{\mathrm{e}}$ determination through He LR spectroscopy shown in the plots are measurement uncertainties only. Systematic uncertainties derived from the atomic model are at the moment not available. They will be assessed in great detail in near future using the Bayesian approach [3].

\subsection{Neon line-ratio spectroscopy}

Injections of $\mathrm{Ne}$ and of mixture $\mathrm{He}+\mathrm{Ne}$ have been carried out in the first and second divertor campaigns to extend the applicability of the diagnostics to the low $T_{\mathrm{e}} /$ high $n_{\mathrm{e}}$ conditions that occur during detached plasmas. These injections were carried out in normal plasma conditions 
(attached plasma) to compare the $T_{\mathrm{e}}$ and $n_{\mathrm{e}}$ determined with LR spectroscopy on Ne and He. The visible-NIR spectrum of Ne I was measured in the region 585 - $880 \mathrm{~nm}$ combining together the $20 \mathrm{~cm}$ and $32 \mathrm{~cm}$ focal length spectrometers along the radial LoS. $20 \mathrm{Ne}$ I lines have been observed in this wavelength range. The $T_{\mathrm{e}}, n_{\mathrm{e}}$ dependence of the line-ratios has been investigated using photon emissivity coefficients from the observed lines calculated with a Ne CRM employing new atomic rate coefficients computed by colleagues from Auburn University [16]. High Rydberg states are at the moment not included in the Ne CRM. Suitable line-ratios have been selected by finding the best agreement between $T_{\mathrm{e}}, n_{\mathrm{e}}$ inferred through $\mathrm{Ne} \mathrm{LR}$ and the ones inferred through He LR without high Rydberg states in comparable discharges (standard magnetic configuration, $\mathrm{P}_{\mathrm{ECRH}}=3 \mathrm{MW}, \bar{n}_{\mathrm{e}}=4.5 \times 10^{19} \mathrm{~m}^{-3}$ ). Line-ratios that show compatible $T_{\mathrm{e}}, n_{\mathrm{e}}$ in the investigated range are $692.9 \mathrm{~nm} / 868.2 \mathrm{~nm}$ for $T_{\mathrm{e}}$ and $703.2 \mathrm{~nm} / 667.8 \mathrm{~nm}$ for $n_{\mathrm{e}}$. Profiles of temperature and density obtained using these ratios are plotted in Figure 4 along with reference profiles from He LR. Temperatures and densities from the Ne LR are about a factor of 2 lower than those computed with the He LR.

Line-ratios from the $\mathrm{Ne}$ lines used to infer $T_{\mathrm{e}}, n_{\mathrm{e}}$ are depicted in Figure 5 for two different $T_{\mathrm{e}}, n_{\mathrm{e}}$ ranges corresponding to attached and detached divertor conditions. We note that these lines might not be the best candidates for application of Ne-beam spectroscopy in the detached divertor conditions $\left(T_{\mathrm{e}}<10 \mathrm{eV}, n_{\mathrm{e}}>10^{20} \mathrm{~m}^{-3}\right)$, since the two line-ratios show both a $T_{\mathrm{e}}$ dependence in this range. The investigation is still in progress and what it is here reported only serves as an example.
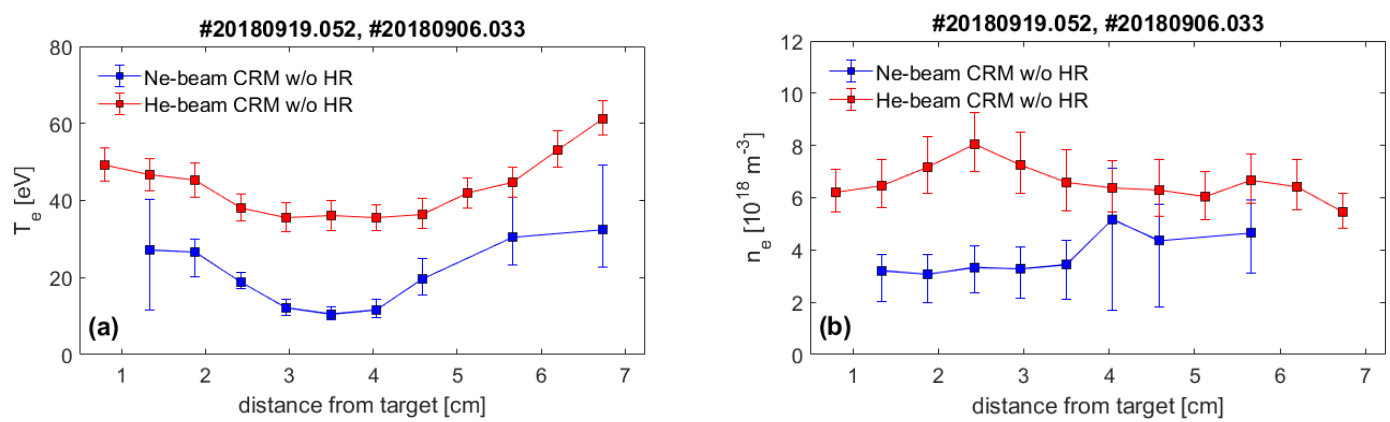

Figure 4. Temperature $(a)$ and density $(b)$ radial profiles obtained from line-ratio spectroscopy on neon and helium in comparable discharges.
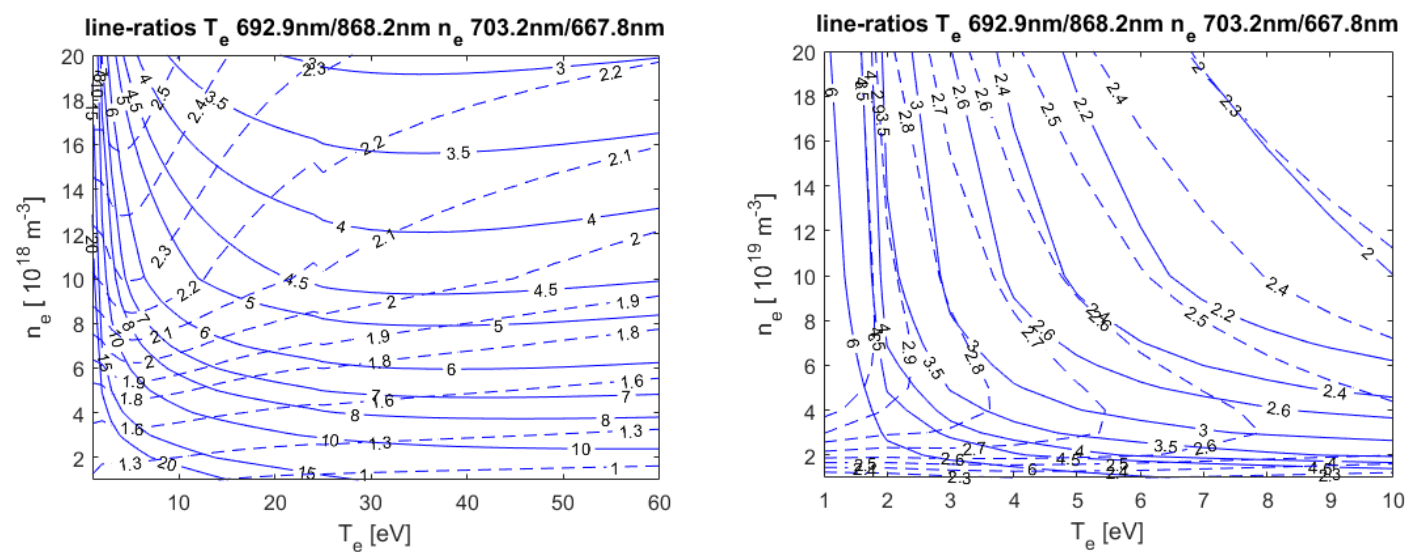

Figure 5. Line-ratios of suitable Ne lines for determining $T_{\mathrm{e}}, n_{\mathrm{e}}$. Solid curves represent the $T_{\mathrm{e}}$-sensitive ratio $692.9 \mathrm{~nm} / 868.2 \mathrm{~nm}$; dashed curves represent the $n_{\mathrm{e}}$-sensitive ratio $703.2 \mathrm{~nm} / 667.8 \mathrm{~nm}$. Two different $T_{\mathrm{e}}, n_{\mathrm{e}}$ ranges are plotted, corresponding to attached (left) and detached (right) divertor conditions. 


\section{Acknowledgments}

This work was funded in part by the U.S. Department of Energy under grant DESC00014210. The publisher, by accepting the article for publication, acknowledges that the United States Government retains a non-exclusive, paid-up, irrevocable, worldwide license to publish or reproduce the published form of this manuscript, or allow others to do so, for United States Government purposes.

This work has been carried out within the framework of the EUROfusion Consortium and has received funding from the Euratom research and training program 2014-2018 and 2019-2020 under grant agreement No 633053. The views and opinions expressed herein do not necessarily reflect those of the European Commission.

\section{References}

1 Schmitz, O. et al. Status of electron temperature and density measurement with beam emission spectroscopy on thermal helium at TEXTOR. Plasma Phys. Control. Fusion, 50, 115004 (2008).

2 Griener, M. et al. Qualification and implementation of line ratio spectroscopy on helium as plasma edge diagnostic at ASDEX Upgrade. Plasma Phys. Control. Fusion, 60, 025008 (2018).

3 Krychowiak, M. et al. Bayesian modelling of a thermal helium beam for measurement of electron density and temperature in the W7-X divertor plasma. Plasma Phys. Control. Fusion, 53, 035019 (2011).

4 Krychowiak, M. et al. Overview of diagnostic performance and results for the first operation phase in Wendelstein 7-X. Rev. Sci. Instrum., 87, 11D304 (2016).

5 Griener, M. et al. Fast piezoelectric valve offering controlled gas injection in magnetically confined fusion plasmas for diagnostic and fuelling purposes. Rev. Sci. Instrum., 88, 033509 (2017).

6 Effenberg, F. et al. Numerical investigation of plasma edge transport and limiter heat fluxes in Wendelstein 7-X startup plasmas with EMC3-EIRENE. Nucl. Fusion, 57, 036021 (2017).

7 www.princetoninstruments.com.

8 Burgos, J. M. Muñoz et al. Hybrid time dependent/independent solution for the He I line ratio temperature and density diagnostic for a thermal helium beam with applications in the scrape-off layer-edge regions in tokamaks. Physics of Plasmas, 19, 012501 (2012).

9 Summers, H. P. et al. Ionisation state, excited populations and emission of impurities in dynamic finite density plasmas: I. The generalized collisional-radiative model for light elements. Plasma Phys. Controlled Fusion, 48, 263 (2006).

10 Drews, P. et al. Edge plasma measurements on the OP 1.2a divertor plasmas at W7-X using the combined probe. Nuclear Materials and Energy, 19 (2019), 179-183.

11 Killer, C. et al. Characterization of the W7-X Scrape-Off Layer using reciprocating probes. Nucl. Fusion, in press (2019).

12 Zoletnik, S. et al. Advanced neutral alkali beam diagnostics for applications in fusion research. Rev. Sci. Instrum., 89, 10D107 (2018).

13 Pasch, E. et al. The Thomson scattering system at Wendelstein 7-X. Rev. Sci. Instrum., 87, 11E729 (2016).

14 Hammond, K.C. et al. Development of a pop-up Langmuir probe array for the W7-X high-heat-flux divertor. (Prague 2018), 45th EPS Conference on Plasma Physics.

15 Zholobenko, W. et al. Synthetic helium beam diagnostic and underlying atomic data. Nucl. Fusion, 58, 126006 (2018).

16 Favreau, C. J. et al. The role of excited-state ionization on the effective ionization of neutral neon using non-perturbative atomic data. J. Phys. B: At. Mol. Opt. Phys., 52, 095203 (2019). 